\title{
Ordered spaces with special bases
}

\author{
by \\ Harold Bennet t (Lubbock, Tex.) \\ and David L u t z er (Williamsburg, Va.)
}

\begin{abstract}
We study the roles played by four special types of bases (weakly uniform bases, $\omega$-in- $\omega$ bases, open-in-finite bases, and sharp bases) in the classes of linearly ordered and generalized ordered spaces. For example, we show that a generalized ordered space has a weakly uniform base if and only if it is quasi-developable and has a $G_{\delta}$-diagonal, that a linearly ordered space has a point-countable base if and only if it is first-countable and has an $\omega$-in- $\omega$ base, and that metrizability in a generalized ordered space is equivalent to the existence of an OIF base and to the existence of a sharp base. We give examples showing that these are the best possible results.
\end{abstract}

1. Introduction. In this paper, we study the roles played by four special types of bases (weakly uniform bases, $\omega$-in- $\omega$ bases, sharp bases, and openin-finite bases) in the classes of linearly ordered and generalized ordered spaces. We characterize ordered spaces with such bases in terms of more familiar topological classes.

To understand the context of our results, recall that many base axioms, known to be distinct in general spaces, seem to fall into three broad equivalence classes when one considers only ordered spaces. First, metrizability in a generalized ordered space $X$ is equivalent to the existence of a $\sigma$-discrete base, or a $\sigma$-locally finite base, or a $\sigma$-locally countable base, or a development for $X$. Second, quasi-developability in a generalized ordered space $X$ is equivalent to the existence of a $\sigma$-disjoint base, or of a $\sigma$-point-finite base. Third, the existence of a point-countable base in a generalized ordered space can be characterized in terms of the Collins-Roscoe property "open (G)" (see $[\mathrm{G}]$ ). (For general information on these topics, see [L].) Our paper shows how the four new base properties mentioned above fit into this pattern.

1991 Mathematics Subject Classification: Primary 54F05, 54D70; Secondary 54E35.

Key words and phrases: point-countable base, weakly uniform base, $\omega$-in- $\omega$ base, open-in-finite base, sharp base, metrizable space, quasi-developable space, linearly ordered space, generalized ordered space. 
We begin Section 2 by characterizing generalized ordered spaces with weakly uniform bases. Heath and Lindgren [HL] defined a collection $\mathcal{C}$ in a space $X$ to be weakly uniform provided that for every pair $x \neq y$ of elements of $X$, the set $\{C \in \mathcal{C}:\{x, y\} \subseteq C\}$ is finite. They noted that a space with a weakly uniform base has a $G_{\delta}$-diagonal, so that in the category of linearly ordered spaces, the existence of a weakly uniform base is equivalent to metrizability. In [HL], the familiar Michael line is cited to show that the analogous assertion fails for generalized ordered spaces. In 2.3 we prove:

1.1. TheOREM. A generalized ordered space has a weakly uniform base if and only if it has a $\sigma$-disjoint base (equivalently, is quasi-developable) and has a $G_{\delta}$-diagonal.

Combined with examples in Section 2, our results show that among generalized ordered spaces, the existence of a weakly uniform base lies strictly between metrizability and quasi-developability. Later, in Corollary 2.7 we show:

1.2. Corollary. Any dense-in-itself generalized ordered space with a weakly uniform base is metrizable.

Also in Section 2 we study the role of OIF and sharp bases among generalized ordered spaces. The notion of an OIF base is due to Balogh who, in a private communication, defined an open-in-finite (OIF) collection to be a collection $\mathcal{C}$ such that if $U$ is any non-empty open subset of $X$, then $U$ is a subset of at most finitely many members of $\mathcal{C}$. It is easy to prove that any metric space, and indeed any metacompact Moore space, has an OIF base, i.e., an OIF collection that is a base for the space, and that a compact Hausdorff space is metrizable if and only if it is first-countable and has an OIF base. Furthermore, Balogh noted that for any $\kappa$, the standard base for the compact space $\{0,1\}^{\kappa}$ is an OIF base. Arkhangel'skiu (see [AJRS]) introduced the notion of a sharp base for a space $X$, namely a base $\mathcal{B}$ for $X$ with the property that if $U_{j}$ is a sequence of distinct members of $\mathcal{B}$, each containing the point $x$, then $\left\{\bigcap\left\{U_{j}: 1 \leq j \leq n\right\}: n \geq 1\right\}$ is a local base at $x$. In 2.6 we show:

1.3. Theorem. For a generalized ordered space $X$, the following are equivalent:

(a) $X$ is metrizable;

(b) $X$ has an OIF base;

(c) X has a sharp base.

In Section 3 we consider a generalization of a point-countable base introduced by Arkhangel'skiǔ, Just, Reznichenko, and Szeptycki [AJRS]: a base 
$\mathcal{B}$ for a space $X$ is an $\omega$-in- $\omega$ base if, for each infinite subset $A \subseteq X$, the collection $\{B \in \mathcal{B}: A \subseteq B\}$ is countable. In 3.3 we prove:

1.4. TheOREM. A linearly ordered topological space has a point-countable base if and only if it is first-countable and has an $\omega-i n-\omega$ base.

That result contrasts sharply with the situation in arbitrary spaces, and even in generalized ordered spaces, as can be seen from 3.1 and 3.4 where we prove:

1.5. EXAMPLE. There exist a linearly ordered space $X$ and a first-countable generalized ordered space $Y$ such that both $X$ and $Y$ have $\omega$-in- $\omega$ bases but neither has a point-countable base. Indeed, both $X$ and $Y$ have a base with the property that only countably many members of the base contain any two distinct points of the space.

However, $\omega$-in- $\omega$ bases do have some interesting consequences in generalized ordered spaces (and in monotonically normal spaces) because in Section 3 we also show that the existence of an $\omega$-in- $\omega$ base for a generalized ordered space (or for a monotonically normal space) guarantees paracompactness.

Recall that a generalized ordered space (GO-space) is a Hausdorff space equipped with a linear order such that the topology has a base whose members are order-convex. Such spaces may be characterized as being those topological spaces that can be embedded in some linearly ordered topological space with its usual open interval topology. A reference for the ordered space notation and terminology used in this paper is $[\mathrm{L}]$. Also recall that a space $X$ is quasi-developable [B] if there is a sequence $\mathcal{G}(n)$ of collections of open subsets of $X$ such that if $U$ is open and $p \in U$, then for some $n$ we have $p \in \operatorname{St}(p, \mathcal{G}(n)) \subseteq U$. Throughout this paper, we will reserve the symbols $\mathbb{R}, \mathbb{P}, \mathbb{Q}$, and $\mathbb{Z}$ for, respectively, the sets of real, irrational, and rational numbers, and the set of integers.

We want to thank K. P. Hart, whose comments significantly shortened and simplified the proofs of our main results.

\section{Weakly uniform, OIF, and sharp bases in GO-spaces}

2.1. LemmA. Let $\mathcal{C}$ be a collection of convex subsets of a GO-space X. Then $\mathcal{C}$ is $\sigma$-star-finite (and hence $\sigma$-point-finite) if either of the following holds:

(a) $\mathcal{C}$ is a weakly uniform collection; or

(b) $\mathcal{C}$ is an OIF collection of open subsets of $X$.

P r o of. First, observe that if $\mathcal{E} \subseteq \mathcal{C}$, then each member of $\mathcal{E}$ is contained in a maximal member (with respect to $\subseteq$ ) of $\mathcal{E}$, because otherwise we could find a strictly increasing sequence $E_{n} \in \mathcal{E}$. But that is impossible in the 
case where $\mathcal{C}$ is weakly uniform (because $E_{2}$ has at least two points and is contained in infinitely many other members of $\mathcal{C}$ ), and also impossible if $\mathcal{C}$ is an OIF collection of open sets.

Let $\mathcal{B}_{0}=\{C \in \mathcal{C}:|C|=1\}$. Clearly, $\mathcal{B}_{0}$ is star-finite (i.e., each member of $\mathcal{B}_{0}$ meets only a finite number of other members of $\left.\mathcal{B}_{0}\right)$. Further, any member of $\mathcal{C}-\mathcal{B}_{0}$ has at least two points. For each $n \geq 0$, let $\mathcal{B}_{n+1}$ be the collection of all maximal members of $\mathcal{C}-\bigcup\left\{\mathcal{B}_{k}: 0 \leq k \leq n\right\}$. We claim that $\mathcal{C}=\bigcup\left\{\mathcal{B}_{k}: k \geq 0\right\}$. For suppose there is a set $C \in \mathcal{C}-\bigcup\left\{\mathcal{B}_{k}: k \geq 0\right\}$. Then $|C| \geq 2$ and for each $k \geq 0, C \in \mathcal{C}-\bigcup\left\{\mathcal{B}_{j}: 0 \leq j \leq k\right\}$. Hence there is a maximal member $B_{k+1}$ of $\mathcal{C}-\left(\mathcal{B}_{0} \cup \ldots \cup \mathcal{B}_{k}\right)$ that contains $C$. The sets $B_{k}$ are clearly distinct, and that is impossible if $\mathcal{C}$ is an OIF collection of open sets, or if $\mathcal{C}$ is a weakly uniform collection. Thus $\mathcal{C}=\bigcup\left\{\mathcal{B}_{k}: k \geq 0\right\}$.

We already know that $\mathcal{B}_{0}$ is star-finite, so fix $k \geq 1$. We begin with a sequence of claims, each easy but somewhat tedious to verify.

Claim 1. If $C_{1}$ and $C_{2}$ are distinct members of $\mathcal{B}_{k}$, then $C_{1}-C_{2}$ and $C_{2}-C_{1}$ are non-empty convex sets.

Claim 2. If $C_{1}$ and $C_{2}$ are distinct members of $\mathcal{B}_{k}$, then every point of $C_{1}-C_{2}$ lies below every point of $C_{2}-C_{1}$, or vice versa, because $C_{1}-C_{2}$ and $C_{2}-C_{1}$ are disjoint convex sets.

For distinct $C_{1}, C_{2} \in \mathcal{B}_{k}$, define $C_{1} \prec C_{2}$ to mean that every point of $C_{1}-C_{2}$ lies below every point of $C_{2}-C_{1}$.

Claim 3. $\prec$ is a linear ordering of $\mathcal{B}_{k}$.

Claim 4. Suppose $C_{1} \prec C_{2}$ in $\mathcal{B}_{k}$ and $p \in C_{1} \cap C_{2}$. Then $\left.C_{1}-C_{2} \subseteq\right] \leftarrow, p[$ and $\left.C_{2}-C_{1} \subseteq\right] p, \rightarrow[$.

Claim 5. Suppose $C_{1} \prec C_{2}$ in $\mathcal{B}_{k}$. Then every point of $C_{1}$ is less than every point of $C_{2}-C_{1}$ and every point of $C_{1}-C_{2}$ is less than every point if $C_{2}$. Consider the assertion for $C_{1}$ and $C_{2}-C_{1}$. Observe that $C_{1}=\left(C_{1}-\right.$ $\left.C_{2}\right) \cup\left(C_{1} \cap C_{2}\right)$. Let $p \in C_{1}$ and $q \in C_{2}-C_{1}$. If $p \in C_{1}-C_{2}$, then $C_{1} \prec C_{2}$ yields $p<q$, and if $p \in C_{1} \cap C_{2}$, then Claim 4 yields $p<q$.

Using those claims about $\prec$, we show that $\mathcal{B}_{k}$ is star-finite. For contradiction, suppose there is a set $B_{0} \in \mathcal{B}_{k}$ such that the collection $\mathcal{D}=\left\{C \in \mathcal{B}_{k}\right.$ : $\left.B_{0} \cap C \neq \emptyset\right\}$ is infinite. Consider $\mathcal{R}=\left\{C \in \mathcal{D}: B_{0} \prec C\right\}$. If $\mathcal{R}$ is infinite, then apply the fact that any infinite linearly ordered set has an infinite strictly monotonic sequence. Choose such a sequence $\left\{D_{n}: n \geq 1\right\}$ in $(\mathcal{R}, \prec)$.

First consider the case where $D_{n} \prec D_{n+1}$ for each $n \geq 1$. Fix points $p \in D_{1}-B_{0}, q \in D_{2}-D_{1}$ and $r \in D_{3}-D_{2}$.

Claim 6. $p<q<r$. Because $p \in D_{1} \cap D_{2}$ and $D_{1} \prec D_{2}$, Claim 4 shows that $\left.q \in D_{2}-D_{1} \subseteq\right] p, \rightarrow\left[\right.$, so that $p<q$. Next, $q \in D_{2}-D_{1} \subseteq D_{2}$ and $r \in D_{3}-D_{2}$, so that applying Claim 5 to $D_{2} \prec D_{3}$ gives $q<r$. 
Claim 7. For each $n \geq 3, r \in D_{n}$. Otherwise, there would be a first $n \geq 3$ with $r \notin D_{n}$. Then $n \geq 4$ and $r \in D_{n-1}-D_{n}$. Because $p \in D_{n} \cap D_{n-1}$, Claim 4 yields $\left.D_{n-1}-D_{n} \subseteq\right] \leftarrow, p$ [ so that $r<p$, and that contradicts Claim 6.

Because each $D_{n}$ is convex, Claim 7 yields $\left.q \in\right] p, r\left[\subseteq[p, r] \subseteq D_{n}\right.$ for each $n \geq 3$. But that is impossible because $\mathcal{C}$ is either weakly uniform or is an OIF collection. (In the weakly uniform case, we have shown that the two points $p$ and $r$ both belong to each $D_{n}$ for $n \geq 3$, and in the OIF case we have shown that the non-empty open set $] p, r$ [ is contained in each $D_{n}$ for $n \geq 3$.)

Next consider the case where $D_{n+1} \prec D_{n}$ for each $n \geq 1$. Then we have $B_{0} \prec D_{n}$ for each $n$ and we choose $p \in B_{0} \cap D_{1}, q \in B_{0} \cap D_{2}$, and $r \in B_{0} \cap D_{3}$. Once again, $\{p, q, r\} \subset D_{n}$ for each $n \geq 3$ and, as above, that is impossible.

Therefore $\mathcal{R}$ is finite. Similarly, $\mathcal{L}=\left\{D \in \mathcal{D}: D \prec B_{0}\right\}$ is finite. Because $\mathcal{D}=\mathcal{L} \cup\left\{B_{0}\right\} \cup \mathcal{R}$, we see that $\mathcal{D}$ is finite. Hence $\mathcal{B}_{k}$ is star-finite.

2.2. Lemma. Let $X$ be a GO-space and suppose $\mathcal{A}$ is either a weakly uniform base or an OIF base. Then the collection $\mathcal{B}$ consisting of all convex components of all members of $\mathcal{A}$ is a base of the same type, and the collection $\mathcal{C}$ of all non-degenerate members of $\mathcal{B}$ is both $\sigma$-star-finite and $\sigma$-locally finite in $X$.

Proof. It is clear that $\mathcal{B}$ is a base for $X$ of the same type as $\mathcal{A}$. Lemma 2.1 shows that $\mathcal{C}=\bigcup\left\{\mathcal{C}_{k}: k \geq 1\right\}$ where each $\mathcal{C}_{k}$ is star-finite in $X$. Thus $\mathcal{C}_{k}$ is locally finite at each point of $\bigcup \mathcal{C}_{k}$. Because $\mathcal{B}$ is a base for $X$, each non-isolated point of $X$ lies in $\bigcup \mathcal{C}_{k}$ for each $k \geq 1$, showing that every point of $X-\cup \mathcal{C}_{k}$ is isolated. Therefore, $\mathcal{C}_{k}$ is locally finite at each point of $X$.

Lemma 2.1 allows us to characterize GO-spaces with weakly uniform bases as follows:

2.3. Proposition. A generalized ordered space $X$ has a weakly uniform base if and only if $X$ is quasi-developable and has a $G_{\delta}$-diagonal.

Pro of. First, suppose that $X$ is quasi-developable and has a $G_{\delta}$-diagonal. Because $X$ is a GO-space and has a $G_{\delta}$-diagonal, $X$ is paracompact [L]. Hence we may assume that $\{\mathcal{G}(n): n \geq 1\}$ is a $G_{\delta}$-diagonal sequence of open covers of $X$ such that $\mathcal{G}(n+1)$ is point-finite in $X$, star-refines $\mathcal{G}(n)$, and consists of convex open subsets of $X$. Because $X$ is quasi-developable, $X$ has a $\sigma$-disjoint base, say $\mathcal{B}=\bigcup\{\mathcal{B}(n): n \geq 1\}$ (see [B], [L]).

For each $B \in \mathcal{B}(n)$, let $\mathcal{H}(B, n)=\{B \cap G: G \in \mathcal{G}(n)\}$ and let $\mathcal{H}(n)=$ $\bigcup\{\mathcal{H}(B, n): B \in \mathcal{B}(n)\}$. Then $\mathcal{H}(n)$ is point-finite and $\mathcal{H}=\bigcup\{\mathcal{H}(n):$ $n \geq 1\}$ is a base for $X$. If $\mathcal{L}=\left\{H_{k}: k \geq 1\right\}$ is an infinite collection of distinct members of $\mathcal{H}$, each containing the point $p$, then point-finiteness 
of each $\mathcal{H}(n)$ forces $\mathcal{L} \cap \mathcal{H}(n) \neq \emptyset$ for infinitely many values of $n$, so that $p \in \bigcap\left\{H_{k}: k \geq 1\right\} \subseteq \bigcap\{\operatorname{St}(p, \mathcal{G}(n)): n \geq 1\}=\{p\}$. Thus, $\mathcal{H}$ is a weakly uniform base for $X$.

To prove the converse, next suppose that $X$ has a weakly uniform base. Heath and Lindgren [HL] have shown that $X$ has a $G_{\delta}$-diagonal. To complete our proof, it will be enough to show that $X$ has a $\sigma$-point-finite base. Let $\mathcal{A}$ be a weakly uniform base for $X$. By 2.2 , the collection of all convex components of members of $\mathcal{A}$ is also a weakly uniform base, and by 2.1 is a $\sigma$-point-finite collection. Thus, $X$ has a $\sigma$-point-finite base, as required.

Our next examples show that the hypotheses of 2.3 cannot be weakened, and that the conclusion of 2.3 cannot be strengthened to metrizability.

2.4. EXAMPLE. There is a LOTS that is quasi-developable and does not have a weakly uniform base, and a GO-space with a weakly uniform base that is not metrizable.

Proof. As shown by Heath and Lindgren [HL], the Michael line $M$ is a GO-space that has a weakly uniform base but is not metrizable. (However, as [HL] noted, a LOTS with a weakly uniform base is metrizable.) To obtain an example of a quasi-developable LOTS that does not have a weakly uniform base, consider the lexicographically ordered set $M^{*}=(\mathbb{R} \times\{0\}) \cup(\mathbb{P} \times \mathbb{Z})$. This linearly ordered topological space is not metrizable (because it contains the Michael line $M$ as a subspace) and therefore cannot have a $G_{\delta}$-diagonal. In the light of [HL], $M^{*}$ cannot have a weakly uniform base. However, $M^{*}$ is known to be quasi-developable [L].

2.5. Remark. There are two covering properties that are naturally associated with the notion of a weakly uniform base, namely that every open cover $\mathcal{U}$ of $X$ has an open refinement $\mathcal{V}$ with the property that if $a$ and $b$ are distinct points of $X$, then $\{V \in \mathcal{V}:\{a, b\} \subseteq V\}$ is finite (respectively, countable). An easy Pressing Down Lemma argument shows that no stationary set in a regular uncountable cardinal can have either of these properties. Consequently, any generalized ordered space, or more generally any monotonically normal space, having either of these two properties must be paracompact ([EL], [BR]).

2.6. TheOrem. For any GO-space $X$, the following are equivalent:

(a) $X$ is metrizable;

(b) $X$ has an OIF base;

(c) X has a sharp base.

Proof. Because any metrizable space has a sharp base, (a) implies (c). Thus it will be enough to show that (c) implies (b) and (b) implies (a). 
To show that (c) implies (b), let $\mathcal{B}$ be any sharp base for $X$. Because the family of all convex components of members of $\mathcal{B}$ is also a sharp base for $X$, we may assume that members of $\mathcal{B}$ are convex. Let $U$ be a non-empty open subset of $X$, and (for contradiction) suppose that there is an infinite collection $\mathcal{C}=\left\{B_{n}: n \geq 1\right\}$ of members of $\mathcal{B}$, each containing the set $U$. If $|U|>1$, choose two points $x \neq y$ of $U$ and note that no finite intersection of members of $\mathcal{C}$ can separate $x$ and $y$, so that the family of finite intersections of members of $\mathcal{C}$ is not a base at $x$. Because $\mathcal{B}$ is a sharp base for $X$, that is impossible. Hence, for some point $p$ we have $U=\{p\}$. Let $\mathcal{D}$ be the family of non-degenerate members of $\mathcal{C}$. Each of the infinitely many members of $\mathcal{D}$ meets either $] \leftarrow, p[$ or $] p, \rightarrow[$. Without loss of generality we may assume that the set $\mathcal{D}^{*}=\left\{B_{i} \in \mathcal{D}: B_{i} \cap\right] \leftarrow, p[\neq \emptyset\}$ is infinite. For each $B_{i} \in \mathcal{D}^{*}$ choose $\left.q_{i} \in B_{i} \cap\right] \leftarrow, p[$. Because $\mathcal{B}$ is a sharp base, there is a $k$ such that $\bigcap\left\{B_{i} \in \mathcal{D}^{*}: 1 \leq i \leq k\right\}=\{p\}$. But that is impossible, because the point $q=\max \left\{q_{i}: i \leq k\right\}$ lies in $\bigcap\left\{B_{i} \in \mathcal{D}^{*}: 1 \leq i \leq k\right\}$ and is less than $p$. That contradiction shows that for any non-void open set $U$, only finitely many members of $\mathcal{B}$ can contain $U$, i.e., that $\mathcal{B}$ is an OIF base for $X$.

To prove that (b) implies (a), let $\mathcal{B}$ be an OIF base for the GO-space $X$. Because of 2.2 we may assume that members of $\mathcal{B}$ are convex, and we know that the collection $\mathcal{C}$ of all non-degenerate members of $\mathcal{B}$ is a base at every non-isolated point of $X$ and is $\sigma$-locally finite in $X$. Next let $I_{k}=\{x \in I: x$ belongs to at most $k$ members of $\mathcal{B}\}$. Then $I_{k}$ is closed in $X$ so that with $\mathcal{I}_{k}=\left\{\{x\}: x \in I_{k}\right\}$, the collection $\bigcup\left\{\mathcal{I}_{k}: k \geq 1\right\}$ is a $\sigma$-discrete collection in $X$ that contains a base at each isolated point of $X$. Thus, $X$ has a $\sigma$-locally finite base and is therefore metrizable.

Theorem 2.6 allows us to obtain a sharpening of Proposition 2.3 for GO-spaces that are dense-in-themselves, namely:

2.7. Corollary. Suppose $X$ is a generalized ordered space that has no isolated points and has a weakly uniform base. Then $X$ is metrizable.

Pr o o f. Let $\mathcal{B}$ be a weakly uniform base for $X$. As in 2.2 , we may assume that members of $\mathcal{B}$ are convex. Any non-empty open set $U \subseteq X$ has at least two points, so that (as in the proof of 2.6) the family of members of $\mathcal{B}$ that contain $U$ must be finite. Hence $\mathcal{B}$ is an OIF base for $X$, so $X$ is metrizable.

3. Point-countable bases in ordered spaces. A collection $\mathcal{C}$ of subsets of a space $X$ is an $\omega$-in- $\omega$ collection provided, for each infinite subset $A \subseteq X$, the collection $\{C \in \mathcal{C}: A \subseteq C\}$ is countable [AJRS]. We begin with an example showing that point-countable collections and $\omega$-in- $\omega$ collections are quite different things, even in a LOTS.

3.1. EXAMPLE. There is a LOTS with an $\omega-i n-\omega$ base that is not firstcountable, and hence does not have a point-countable base. 
Proof. Let $\mathbb{Z}$ be the usual set of integers, and let $X$ be the lexicographically ordered set $\left(\left[0, \omega_{1}[\times \mathbb{Z}) \cup\left\{\left(\omega_{1}, 0\right)\right\}\right.\right.$ endowed with the usual open interval topology of that order. Write $\Omega=\left(\omega_{1}, 0\right)$ and let $\mathcal{B}=\{\{p\}: p \in$ $X, p<\Omega\} \cup\{] p, \Omega]: p<\Omega\}$. Clearly, $\mathcal{B}$ is a base for $X$. Then $\mathcal{B}$ is an $\omega$-in- $\omega$ base for $X$. In fact we will show more. Let $A$ be any subset of $X$ with at least two points. Let $\alpha$ be the least of the first coordinates of points in $A$. Then $\alpha<\omega_{1}$, so that $A$ cannot be a subset of any $\left.] p, \Omega\right] \in \mathcal{B}$ where the first coordinate of $p$ is above $\alpha$. Thus, $A$ is a subset of at most countably many members of $\mathcal{B}$.

The situation in a first-countable LOTS contrasts sharply with 3.1, as can be seen from:

3.2. Proposition. Let $X$ be a first-countable LOTS and let $\mathcal{C}$ be an $\omega$-in- $\omega$ collection of convex open subsets of $X$. Then $\mathcal{C}$ is a point-countable collection.

Proof. For $p \in X$, let $\mathcal{C}(p)=\{C \in \mathcal{C}: p \in C\}$. We will show that each $\mathcal{C}(p)$ is countable. First consider the case where $p$ is not isolated. Then there is a sequence $x_{k}$ of distinct points of $X$ that converges to $p$. Let $A_{n}=\left\{x_{k}: k \geq n\right\}$ and observe that if $C \in \mathcal{C}(p)$, then $A_{n} \subseteq C$ for some $n$. Because $\mathcal{C}$ is an $\omega$-in- $\omega$ collection, each $\mathcal{C}_{n}=\left\{C \in \mathcal{C}: A_{n} \subseteq C\right\}$ is countable. Because $\mathcal{C}(p) \subseteq \bigcup\left\{\mathcal{C}_{n}: n \geq 1\right\}, \mathcal{C}(p)$ is countable whenever $p$ is a non-isolated point of $X$.

Next we show that $\mathcal{C}(p)$ is countable in case $p$ is an isolated point of $X$. Let $X^{+}$denote the usual Dedekind compactification of $X$. For the rest of this proof, interval notation will refer to intervals in $X^{+}$. Each member $C \in \mathcal{C}(p)$ has the form $C=] u_{C}, v_{C}\left[\cap X\right.$ where $u_{C}, v_{C} \in X^{+} \cup\{\leftarrow, \rightarrow\}$. Consider the set $R=\left\{v_{C}: C \in \mathcal{C}(p)\right\}$. We will show that $R$ is countable.

In case the set $[p \rightarrow[\cap X$ is finite, so is $R$. Consider the case where $\left[p \rightarrow\left[\cap X\right.\right.$ is infinite. Then $p$ is not the right end point of $X$. Let $p_{0}=p$. Because $p_{0}$ is an isolated point of the LOTS $X$, there is a point $p_{1} \in X$ with $p_{0}<p_{1}$ and $] p_{0}, p_{1}\left[=\emptyset\right.$. We will say that $p_{0}$ and $p_{1}$ are adjacent points. For induction, suppose $n \geq 1$ and that we have defined a strictly increasing sequence $p_{j}$ of adjacent points for $0 \leq j \leq n$. If $p_{n}$ is a limit point of $X$, we define $q=p_{n}$ and the induction stops. If $p_{n}$ is not a limit point of $X$, then there is a point $p_{n+1}$ that is the immediate successor of $p_{n}$ in $X$ (because $p_{n}$ cannot be the right end point of $X$ since $[p \rightarrow[\cap X$ is infinite), and the induction continues. If $p_{n}$ is defined for each $n \geq 0$, then let $q=\sup \left\{p_{n}: n \geq 0\right\}$, the supremum being taken in the compact space $X^{+}$. In either case, we have a limit point $q \in X^{+}$such that $p<q$ and $[p, q]$ is countable.

Let $\left.\left.R_{1}=R \cap\right] p, q\right]$ and $\left.R_{2}=R \cap\right] q \rightarrow\left[\right.$. Then $R=R_{1} \cup R_{2}$. Clearly, $R_{1}$ is countable, so it is enough to show that $R_{2}$ is also countable. Fix $v_{C} \in R_{2}$. 
Then $] u_{C}, v_{C}[\cap X \in \mathcal{C}(p)$ and $[p, q] \subset] u_{C}, v_{C}[$. There are two cases to consider. If $q \in X$, then $v_{C} \in R_{2}$ implies $] u_{C}, v_{C}[\cap X \in \mathcal{C}(q)$ and the first part of the proof shows that $\mathcal{C}(q)$ is countable because $q$ is not isolated. If $q \in X^{+}-X$, then the set $S=X \cap[p, q[$ is infinite so that because $\mathcal{C}$ is an $\omega$-in- $\omega$ collection, the family $\mathcal{C}(S)=\{C \in \mathcal{C}: S \subseteq C\}$ is countable. Observe that $v_{C} \in R_{2}$ implies $C=(] u_{C}, v_{C}[\cap X) \in \mathcal{C}(S)$. Therefore $R_{2}$ is countable. Hence $R$ is countable.

A similar argument shows that $L=\left\{u_{C}: C \in \mathcal{C}(p)\right\}$ is also countable. Hence so is $\mathcal{C}(p)$, as required.

We want to thank the referee for pointing out an error in a draft version of this paper in which 3.2 was asserted to hold for collections of open sets that are not necessarily convex. As the referee noted, an uncountable almost disjoint collection of infinite subsets of the usual space of integers is an $\omega$-in- $\omega$ collection of open sets in a first-countable LOTS that is not point-countable.

3.3. Corollary. A LOTS $X$ has a point-countable base if and only if $X$ is first-countable and has an $\omega$-in- $\omega$ base.

P r o o f. First, suppose that $X$ has an $\omega$-in- $\omega$ base $\mathcal{C}$. If necessary, replace $\mathcal{C}$ by the collection $\mathcal{C}^{\prime}$ of all convex components of all members of $\mathcal{C}$, and observe that if $\mathcal{C}$ is an $\omega$-in- $\omega$ collection, then so is $\mathcal{C}^{\prime}$. Further, observe that because $\mathcal{C}$ is a base for $X$, so is $\mathcal{C}^{\prime}$. Apply 3.2 to conclude that $\mathcal{C}^{\prime}$ is a point-countable base for $X$. The converse is clear.

The role of $\omega$-in- $\omega$ bases in generalized ordered spaces, as opposed to LOTS, is more complicated. We begin with an example showing that a first-countable GO-space can have an $\omega$-in- $\omega$ base without having a pointcountable base. The example is a variation on the space known as the "Big Bush" [B2].

3.4. EXAMPLE. There is a first-countable GO-space $X$ that has an $\omega$-in- $\omega$ base, but does not have a point-countable base.

Pr o of. Let $\mathbb{R}, \mathbb{P}$, and $\mathbb{Q}$ denote the sets of real, irrational, and rational numbers, respectively. Let $X$ be the set of all functions $f:\left[0, \omega_{1}[\rightarrow \mathbb{R}\right.$ with the property that either $f(\alpha) \in \mathbb{P}$ for all $\alpha<\omega_{1}$ or else there is a $\beta<\omega_{1}$ such that $f\left[\left[0, \beta[] \subseteq \mathbb{P}\right.\right.$ and $f(\gamma)=f(\beta) \in \mathbb{Q}$ for each $\gamma \in\left[\beta, \omega_{1}[\right.$. In the first case we will write $L(f)=\omega_{1}$ and in the second $L(f)=\beta$. Lexicographically order the set $X$, i.e., if $f \neq g$ are points of $X$, let $\gamma$ be the first ordinal where $f(\gamma) \neq g(\gamma)$, and define $f<g$ to mean that, in $\mathbb{R}, f(\gamma)<g(\gamma)$. This linear ordering induces an open interval topology on $X$, and we modify that topology by declaring that every point $f$ with $L(f)=\omega_{1}$ is isolated. It is easy to see that the resulting GO-space is first-countable.

Suppose that $f \in X$ and $L(f)<\omega_{1}$. For each $n \geq 1$ define two new functions $f_{-n}$ and $f_{+n}$ by 


$$
\begin{aligned}
& f_{-n}(\gamma)= \begin{cases}f(\gamma) & \text { if } \gamma<L(f), \\
f(\gamma)-1 / n & \text { if } L(f) \leq \gamma<\omega_{1},\end{cases} \\
& f_{+n}(\gamma)= \begin{cases}f(\gamma) & \text { if } \gamma<L(f), \\
f(\gamma)+1 / n & \text { if } L(f) \leq \gamma<\omega_{1}\end{cases}
\end{aligned}
$$

It is easy to verify that:

(a) In $X$, if $g<h<k$ and if $g(\beta)=k(\beta)$ for each $\beta<\alpha$, then $h(\beta)=$ $g(\beta)$ for each $\beta<\alpha$.

Consequently,

(b) In $X$ if $\alpha=L(f)<\omega_{1}$, then $] f_{-n}, f_{+n}[=\{g \in X: g(\beta)=f(\beta)$ if $\beta<\alpha$ and $|f(\alpha)-g(\alpha)|<1 / n\}$; and

(c) The sets $B(f, n)=] f_{-n}, f_{+n}[$ form a neighborhood base at $f$ whenever $L(f)<\omega_{1}$.

Let

$$
\begin{aligned}
\mathcal{B}=\{ & \left.B(f, n): f \in X \text { and } L(f)<\omega_{1} \text { and } n \geq 1\right\} \\
& \cup\left\{\{f\}: f \in X \text { and } L(f)=\omega_{1}\right\} .
\end{aligned}
$$

Clearly, $\mathcal{B}$ is a base for $X$. We claim that $\mathcal{B}$ is an $\omega$-in- $\omega$ collection. We will prove much more, namely that if $f \neq g$ are points of $X$, then only countably many members of $\mathcal{B}$ contain $\{f, g\}$. To that end, let $\gamma$ be the first ordinal where $f$ and $g$ differ. Then $\gamma<\omega_{1}$. Suppose $B \in \mathcal{B}$ contains both $f$ and $g$. Then $B$ cannot be a singleton, so $B$ must have the form $B(h, n)$ for some $h \in X$ with $L(h)<\omega_{1}$ and some $n \geq 1$. We will complete this part of the proof by showing that there are only countably many possible functions $h$ with $\{f, g\} \subseteq B(h, n)$. First note that $L(h) \leq \gamma$. Next observe that the values of $h$ on $[0, L(h)$ [ coincide with the values of $f$ on $[0, L(h)[$ and that there are only countably many possibilities for the rational number $h(L(h))$. Because $\gamma<\omega_{1}$, we now see that $\{B \in \mathcal{B}:\{f, g\} \subseteq B\}$ is a countable collection.

It only remains to observe that no base for the space $X$ can be pointcountable. The proof parallels the argument given in [B2] or [BLP].

Even though the existence of an $\omega$-in- $\omega$ base does not yield a pointcountable base in a GO-space, such bases do have a role to play because they guarantee paracompactness in a GO-space, and in the more general category of monotonically normal spaces. We begin with:

3.5. LemMA. Let $S$ be a stationary set in an uncountable regular cardinal $\kappa$. Then the open cover $\mathcal{U}=\{[0, \alpha[\cap S: \alpha \in S\}$ has no open refinement that is an $\omega$-in- $\omega$ collection.

Pr o of. For contradiction, suppose there is an open refinement $\mathcal{V}$ of $\mathcal{U}$ that is an $\omega$-in- $\omega$ collection. Let $T$ be the set of non-isolated points of the subspace $S$ of $[0, \kappa[$. Then $T$ is also stationary in $\kappa$. Because $\mathcal{V}$ refines $\mathcal{U}$, for 
each $\lambda \in T$ we may choose $V(\lambda) \in \mathcal{V}$ and $\alpha(\lambda)>\lambda$ such that $\lambda \in V(\lambda) \subseteq$ $[0, \alpha(\lambda)[\cap S \in \mathcal{U}$. Observe that if $\lambda<\mu$ are points of $T$ with $\alpha(\lambda)<\mu$, then $V(\lambda) \neq V(\mu)$. Choose $\beta(\lambda)<\lambda$ such that $S \cap] \beta(\lambda), \lambda] \subseteq V(\lambda)$. The Pressing Down Lemma yields a point $\gamma$ and a cofinal set $T^{\prime} \subseteq T$ such that $\beta(\lambda)=\gamma$ for each $\lambda \in T^{\prime}$. Choose $\delta \in S$ such that the set $A=S \cap[\gamma, \delta]$ is infinite. Because $\kappa$ is regular and uncountable, there are uncountably many $\lambda \in T^{\prime}$ with $\lambda>\delta$. But then $A$ is a subset of uncountably many distinct sets $V(\lambda) \in \mathcal{V}$, and that is impossible.

3.6. Corollary. A monotonically normal space $X$ is paracompact if and only if every open cover of $X$ has an open refinement that is an $\omega$-in$\omega$ collection. In particular, this characterization applies to any generalized ordered space.

P r o of. By a result of Balogh and Rudin [BR], if a monotonically normal space is not paracompact, then it contains a stationary subset of a regular uncountable cardinal as a closed subspace. Now apply 3.5.

\section{References}

[AJRS] A. Arkhangel'skiǔ, W. Just, E. Reznichenko and P. Szeptycki, Sharp bases and weakly uniform bases versus point countable bases, Topology Appl., to appear.

[BR] Z. Balogh and M. E. Rudin, Monotone normality, ibid. 47 (1992), 115-127.

[B] H. Bennett, On quasi-developable spaces, Gen. Topology Appl. 1 (1971), 253262.

[B2] - , Point-countability in linearly ordered spaces, Proc. Amer. Math. Soc. 28 (1971), 598-606.

[BLP] H. Bennett, D. Lutzer, and S. Purisch, On dense subspaces of generalized ordered spaces, Topology Appl., to appear.

[EL] R. Engelking and D. Lutzer, Paracompactness in ordered spaces, Fund. Math. 94 (1976), 49-58.

[G] G. Gruenhage, A note on the point-countable base question, Topology Appl. 44 (1992), 157-162.

[HL] R. Heath and W. Lindgren, Weakly uniform bases, Houston J. Math. 2 (1976), 85-90.

[L] D. Lutzer. On generalized ordered spaces, Dissertationes Math. 89 (1971).

Texas Tech University

Lubbock, Texas 79409

U.S.A

E-mail: graddir@math.ttu.edu
College of William and Mary Williamsburg, Virginia 23187 U.S.A.

E-mail: lutzer@math.wm.edu 